\title{
Green Computing: Issues on the Primary Memory of Personal Computers
}

\author{
A. Mala, C. Umarani and L. Ganesan \\ Faculty from A C College of Engineering \& Technology, Karaikudi-630 004, TN IN
}

\begin{abstract}
Green computing is now a days becoming a research problem and many scientists are focusing their attention to do research on various issues related to this discipline. The topic addresses issues such as, effective power utilization, maximizing efficiency, maximizing efficiency of utilization, correct disposal of electronic gadgets, reliable and cost effectiveness etc. One of the most used components by ICT group are personal computers and hence considered for our analysis under green computing. Any personal computer has major functional components such as CPU, memory, monitors and peripheral devices etc. Each components have certain contribution towards green computing. The main focus of attention of this paper is to study how the power consumed by each functional units are effectively minimized so as to perform the job without any deviation while using the personal computer. Power thus saving is equivalent to power generation or optimal usage. Since there are many functional units present in a personal computer, in this paper, we discuss how memory is contributing to the green computing by conducting various studies on SDRAMs. Results and discussions are presented. The work related to the monitor and the processors are in progress.
\end{abstract}

Keywords: Green computing - Personal computers - Effective power utilization - Contribution from memory Variations of power consumption at different frequencies.

\section{Introduction}

Green computing is a technology that is now under attention of business, researchers, and industries for the energy efficiency. It is realized that going green is in best interest, both in terms of public utilization and reduced costs.

Computing was focused initially on faster analysis and speedier calculation and solving of more complex problems in a shorter time with good accuracy . But in the recent past Green computing has gained importance for achieving of energy efficiency, minimization of power consumption of any equipment. The main objective of Green computing technology is to study and practice computing resources efficient and ecofriendly. Maximizing the energy efficiency is one of the primary focus of this technology.

Green computing is required to make our self and our environment healthy. It can be defined as responsibly utilizing the resources available. Many computers are produced from many hazardous materials like cadmium, mercury and other toxic substances. While disposing the computers, it will lead to pollution and affect the environment to a great extent. This field encompasses a broad range from new generation techniques to the study of advanced materials to be used in daily life.

The impact of the toxic wastes that are produced by us through throwing our old computers and peripherals lead to land pollution. The computers have the power hogs that generate pollution by the energy they consume for their processes.

Green computing addresses many problems. To be in precise, the goals are (which are collected from various literatures as mentioned in reference,[1-4]).

$>$ To reduce the power consumption of the products

$>$ To reduce the harmful effects to the environments through the use of hazardous materials

$>$ To reduce the carbon foot prints

$>$ To increase the life time of the product

$>$ To maximize energy efficiency during the product's lifetime

$>$ To promote recyclability of defunct products and minimize factory waste

Power dissipation is also a major concern in portable, battery-operated devices. Each of us has experienced the event that the battery of our laptop or mobile phone is depleted. Finally, energy dissipation causes thermal problems. Most of the energy consumed by a system is converted into heat, resulting in wear and reduced reliability of hardware components.

In a personal computer, many hardware components are used. To name a few, CPU, processor, memory, input and output or peripheral devices etc. Each one is contributing for the computation and delivery of output. The power consumed by each component has to be maximally converted in to useful outcome. In this study, our main focus is on the effective utilization power for primary memory which is attached with a 
personal computer system. For every micro operations performed in the system, the memory unit is referred. Typically if we look at the power consumed by the sum of power handled by each functional components such as Processor, monitor, hard disk, DVD, VGA, PCI, motherboard, RAM and peripherals. Among these eight parameters, we have considered only about memory. For a Celeron and Pentium IV processors, the power consumed by various components are illustrated in the following pie charts. The peripherals and the functional units used in both the processors are given below the chart. The major components which are consuming more power are the processor, monitor and RAM. Of course the other units consume more or less same power. The pie charts are drawn using the details of the processors collected from the literature [6].
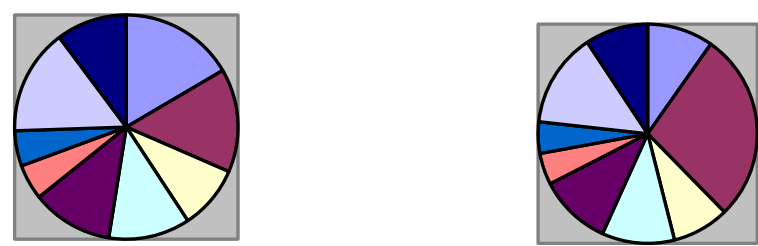

\begin{tabular}{|l|}
\hline QMonitor \\
QProcessor \\
QHard Disk \\
QDVD \\
QVGA \\
QPCI \\
QMother Board \\
QRAM \\
Peripherals \\
\hline
\end{tabular}

Figure 1. Pie Charts for the Power consumption in Celeron and P-IV based systems

The above figure is drawn for the processors Celeron and the Intel P-IV whose details are given below.

Celeron Conroe - $\mathrm{L}(65 \mathrm{~nm})$

MONITOR
HARD DISK
VGA
MOTHER BOARD
DVD
RAM
PCI

PERIPHERALS

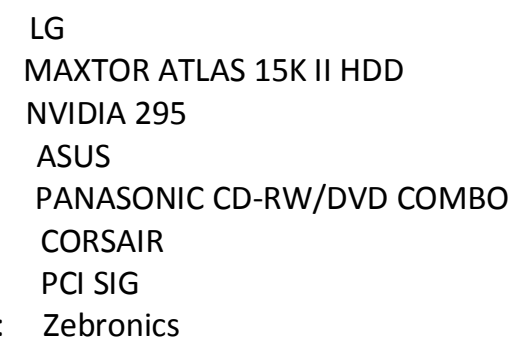

LG

MAXTOR ATLAS 15K II HDD

NVIDIA 295

ASUS

PANASONIC CD-RW/DVD COMBO

CORSAIR

PCI SIG

Zebronics

Pentium -IV ( Northwood)

LG LED 20inch (50.8 CMS) E51
MAXTOR ATLAS 15K II HDD
NVIDIA 295
ASUS
PANASONIC CD-RW/DVD COMBO
CORSAIR
PCI SIG
Zebronics

Our idea is to present various implications of memory in a personal computer in Green computing perspective. The organization of the paper is as follows. Second section of this paper discusses about the proposal of the work and third section presents the methodology and the calculations. Fourth section presents the results and discussion. Finally fifth section illustrates the conclusion and further scope for the present work.

\section{PROPOSAL}

Saving power is the main objective of Green Computing and that is also with minimum impact to the environment. It is about how to reduce the power consumption of these memory devices to save energy, increase the life time of the product and also to make it efficient.

"Faster processors use more power, because they use too much power and their waste heat increases temperature for which air conditioning necessary, especially in server farms--between the computers and the HVAC. The waste heat also causes reliability problems, as CPU's crash much more often at higher temperatures"[5].

A bus is simply a circuit that connects one part of the motherboard to another. The more data a bus can handle at one time, the faster it allows information to travel. The speed of the bus, measured in megahertz $(\mathrm{MHz})$, refers to how much data can move across the bus simultaneously. Bus speed usually refers to the speed of the front side bus (FSB), which connects the CPU to the north bridge. Since the CPU reaches the memory controller though the north bridge, FSB speed can dramatically affect the performance of the computer.

Over clocking is the act of increasing the speed of certain components in a computer other than that specified by the manufacturer. It mainly refers to making CPU run at a faster rate although it could also refer to making the graphics card or other peripherals run faster.

When increased, it makes the whole system run faster and not only the CPU. Most of the PC's have a speed of $66 \mathrm{MHz}$ or $100 \mathrm{MHz}$. Mainly, it defines the speed that the motherboard and other components run at. Multipliers are located on the motherboard and come in the forms $\mathrm{x} 1, \mathrm{x} 1.5, \mathrm{x} 2, \mathrm{x} 2.5$, etc...called as Jumpers. When the Bus speed is multiplied by any one of these numbers, it results in the final CPU speed. Our attention is focused to memory in general, SDRAM in particular. 
SDRAM (Synchronous Digital Random Access Memory) is the main internal memory that connects directly to the motherboard. Its main function is to temporarily store data to aid the processor (CPU) in computations. The internal memory has associated with a preset frequency rate. The clock speed of this memory can be increased in the BIOS settings (Basic Input / Output System), which is a basic operating system that controls all hardware attached to the computer. This process of manually setting the SDRAM frequency is called "over clocking."

By varying this SDRAM frequency, the bus speed can be changed which in turn changes the clock speed. The variation in the clock speed by SDRAM frequency can be calculated from the jumper value (the number of pins configured from mother board) multiplied with it. Through this, we can calculate the change in power consumption of the CPU with respect to the clock speed.

Now our proposal is to change this frequency for SDRAM unit to make corresponding changes in the clock speed and measure the power consumption of the CPU at that instance. This SDRAM frequency is available in the Pentium 4 processor which will control the Front side bus (FSB) of the system. When the FSB is controlled, we can control the number of instructions that are fetched to the processor for execution. So the number of instructions that will be executed get reduced which in turn reduces the work load of the processor and the heat consumption of the processor.

\section{Methodologies \& Procedures}

In the previous section, how the SDRAM frequency influences the CPU power and hence affects the overall power handling. In this section, the procedure for carrying out the experimentation are illustrated.

For our experimentation, a Pentium - IV system is considered. From the SMPS, various units are powered. Using the measuring devices, the voltage and the current are measured and are used for the calculations. SDRAM frequency which mainly influences the clock speed is altered and power consumption of the system with a Pentium 4 processor is measured. As it is discussed in the previous section, it influences the Bus speed directly which in turn changes the clock speed of the processor.

Normally the clock speed is calculated by the product of Bus speed and Clock multiplier It's the pin connection coming out of the processor.

Clock speed $=$ Bus speed $($ SDRAM frequency) $*$ an integer value (Jumper value)

SMPS (Switched Mode Power Supply) : The power to the different parts of the computer is distributed from the Switched Mode Power Supply only. It distributes power to all parts of the computer. It converts the AC in to DC, and then sends it all across the computer. It provides the power supply to the processor and mother board in different required voltages.

Central Processing Unit : $\quad$ The CPU power splits up in to various functional units Power(CPU) $=$ Power taken by (Video card + Mother board + Hard drive + CD/DVD drive + CPU fan + RAM + Processor + Optional (or peripheral) devices) which are described as follows.

Video card: A video card (also otherwise called a video adapter, display card, graphics card, graphics board, display adapter or graphics adapter) is an expansion card which generates a feed of output images to a display. Most video cards offer various functions such as accelerated rendering of 3D scenes and 2D graphics, MPEG-2/MPEG-4 decoding, TV output, or the ability to connect multiple monitors. In general, video card consumes $30-50$ watts.

Mother Board: A mother board is the main circuit board in a system. The power consumed by mother board is not directly by it, it is consumed by the ports and peripherals connected with it.

Hard Drive: A hard drive is the main storage component in a system. It is a magnetic disc used to store data and also called as main memory.

CD/DVD Drive: A CD/DVD drive is the external storage component of a computer. It is called as the secondary memory.

RAM: Random-access memory (RAM) is a form of computer data storage. A random-access device allows stored data to be accessed quickly in any random order. It is called as the primary memory in a memory.

Processor: The processor is the heart of the computer. It controls the function of all parts of the computer. It is operated with a fan to reduce the heat generated during a process. A processor will consume $80-140$ watts.

The experimentation is started, in a Pentium 4 Processor of a PC. It has the SDRAM frequency, at the maximum of $400 \mathrm{MHz}$ with a clock speed of $2.4 \mathrm{GHz}$. It can be varied in the Basic Input and Output System (BIOS) during the startup of the operating system using F2 key.

In Pentium 4 processor, it possess SDRAM frequency of the following values.

$\checkmark \quad 400 \mathrm{MHZ}$ 


\section{$\checkmark \quad 333 \mathrm{MHZ}$}

$\checkmark \quad 266 \mathrm{MHZ}$

In Pentium 4 processor, the power supply from the SMPS (Switched Mode Power Supply) consists of many wires carrying DC power connections. These carry $+5 \mathrm{~V},-5 \mathrm{~V},+12 \mathrm{~V},-12 \mathrm{~V}$ and a power good signal. The power good signal is a special flag indicating that the supply are stable and usable. To measure the voltage drop, a multi meter is used in two sliced ends of the wires. The maximum value displayed by the ammeter is considered when it's connected with the processor's power supply for measuring the current consumed by the unit from the SMPS.

\section{Procedure}

$>$ The power to the processor is provided from SMPS through two yellow wires, each of 12 Volts.

$>$ The power connector of the processor consist of a four pin structure which is connected with the power supply of the processor

$>$ In that the two yellow wires, will provide supply to the processor in which the current varies.

$>$ Those two wires are serialized with the ammeter to measure the current varying in it

$>$ The computer's SDRAM frequency can be varied only using the BIOS of the system

$>$ In BIOS, the SDRAM frequency is varied by selecting "Advanced Settings".

$>$ In Advanced settings, "chip set configuration" should be selected and in that the SDRAM frequency should be selected and changed.

\section{Results \& Discussions}

For the experimentation, it is very important to create the conditions suitable for performing. Enable the BIOS settings before measuring the power from the SMPS to the processor. The SDRAM frequency has to be adjusted before doing any measurements.

IDLE: This is the state when the computer is not doing any work and the processor is not at all working.

ACTIVE: The active state is the state in which the CPU usage is at $100 \%$. That kind of environment is created by rendering a video clip in the system. Rendering usually consumes CPU at higher level which makes it possible to measure power at this stage.

\subsection{Power Analysation}

\section{IDLE:}

\section{1) At $400 \mathrm{MHz}$}

Voltage $=12.56 \mathrm{~V} \quad \mathrm{P} 1=1.4 \mathrm{~A} \quad \mathrm{P} 2=1.0 \mathrm{~A}$

Total Power $=12.56+17.58=30.144$ Watt.

2) At $333 \mathrm{MHz}$

Voltage $=12.56 \mathrm{~V} \quad \mathrm{P} 1=0.7 \mathrm{~A} \quad \mathrm{P} 2 \quad=1.2 \mathrm{~A}$

Total Power $=15.072+8.792=23.864$ Watt.

3) At $266 \mathrm{MHz}$

Voltage $=12.56 \mathrm{~V}$ P1 $=0.7 \mathrm{~A} \mathrm{P} 2=0.6 \mathrm{~A}$

ACTIVE:

Total Power $=8.792+7.536=16.328$ Watt.

\section{1) At $400 \mathrm{MHZ}$}

Voltage $=12.56 \mathrm{~V} \quad \mathrm{P} 1=3.2 \mathrm{~A} \quad \mathrm{P} 2=2.6 \mathrm{~A}$

Total Power $=40.192+32.656=72.848$ Watt.

Table : 1 Power computation against Frequency variation

\begin{tabular}{|l|l|l|l|l|l|}
\hline \multirow{2}{*}{ Frequency } & \multicolumn{2}{|c|}{$\mathrm{V}$} & \multicolumn{2}{|c|}{ P1 } & \multicolumn{2}{|c|}{ P2 } & \multicolumn{2}{c|}{ Power utilization } \\
\cline { 4 - 6 } & & & & Idle & Active \\
\hline $400 \mathrm{MHz}$ & 12.56 & $1.4 \mathrm{~A}$ & $1.0 \mathrm{~A}$ & 30.144 & 72.84 \\
\hline $333 \mathrm{MHz}$ & 12.56 & $0.7 \mathrm{~A}$ & $1.2 \mathrm{~A}$ & 23.864 & \\
\hline $266 \mathrm{MHz}$ & 12.56 & $0.7 \mathrm{~A}$ & $0.6 \mathrm{~A}$ & 16.328 & \\
\hline
\end{tabular}

From the above result, it is observed that the SDRAM frequency influences the power consumption of the processor by varying the clock speed. As the SDRAM frequency changes SDRAM frequency and the power consumption is also increased. So whenever necessary and possible, the SDRAM frequency can be as desired to keep power consumption less. The experiments are repeated for various types of processors and the 
power consumption is noted. The results are displayed in Table 1 and the corresponding graph is plotted and is shown in figure2.

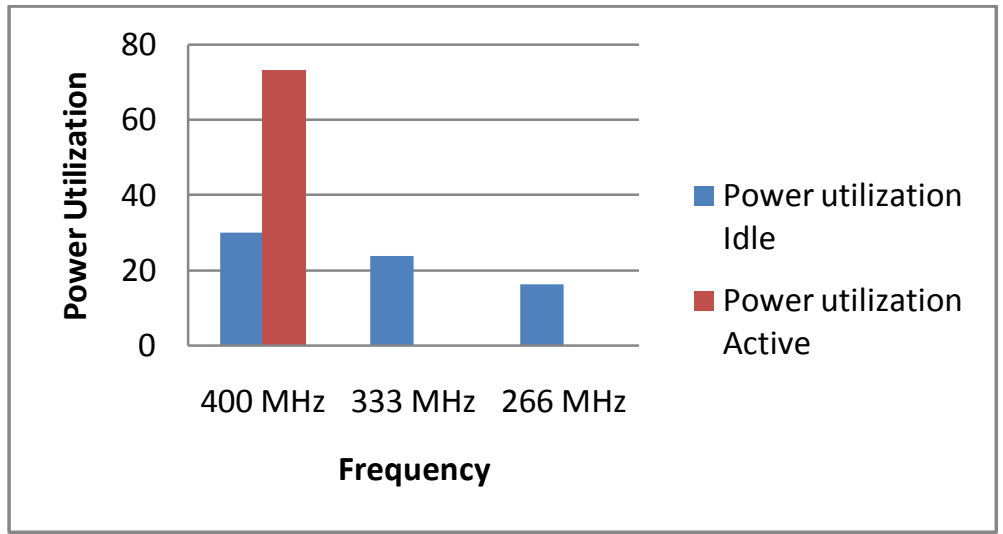

Figure 2: The graph shows variation of frequency Vs Power

\section{Conclusions}

The SDRAM frequency is varied and the corresponding changes in the power consumption of processor is measured from the power plug of the processor using the above procedure. The suitable environment for the experimentation is thus created and the power distributed from SMPS is measured successfully. The variation of the speed with request to the SDRAM frequency is thus observed. It shows when the SDRAM memory frequency is adjusted, the power consumption can be saved. The influence of the memory is inferred from the above observations. Every intuition says for a given problem, the use of less power is preferred. What type of problem it is, when the problem is supposed to be completed and what is the clock the memory unit can be set so that the power drawn from the SMPS is lowered and enables green computing.

\section{References:}

[1]. Priya Rana, "Green Computing Saves Green", Department Of Information Technology, RKGIT, Ghaziabad International Journal Of Advanced Computer And Mathematical Sciences. Vol 1, Issue 1, Dec, 2010, Pp 45-51.

[2]. Er. Navdeep Kochhar and Er. Arun Garg, "Eco-friendly Computing: Green Computing", Baba Farid College, Bathinda, Punjab. International Journal of Computing and Business Research,ISSN (Online) : 2229-6166, Volume 2 Issue 2 May 2011.

[3]. Robert R. Harmon and Nora Auseklis, "Sustainable IT Services: Assessing the Impact of Green Computing Practices". Portland State University, Strategic Marketing Area, Portland, OR, USA Intel Corporation, Engineering computing, Hillsboro, OR, USA. PICMET 2009 Proceedings, August 2-6, Portland, Oregon USA @ 2009 PICMET.

[4]. Enrico Bini, Giorgio Buttazzo and Giuseppe Lipari, "Minimizing CPU Energy in Real-Time Systems with Discrete Speed Management". Scuola Superiore Sant'Anna, Pisa, Italy.

[5]. Susanne Albers, "Energy efficient algorithms", Communications of the ACM, Vol. 53, No. 5, pp. 86-96.

[6]. Intel® PentiumR 4 Processor or $90 \mathrm{~nm}$ Process. Datasheet Document No.: 300561-003. 